\title{
BERICHT
}

\section{Eine linguistische Wende in der Logik?}

Bericht über den 7. Internationalen Kongreß für Logik, Methodologie und Wissenschaftstheorie vom 11.-16. Juli 1983 in Salzburg

\section{GERHARD HEYER}

\section{Summary}

Reporting on the 7th International Congress of Logic, Methodology, and Philosophy of Science, first the main topics and some organisational aspects of the congress are presented; the main part of the report focuses on recent developments in Philosophical Logic (Section 5), in particular the theory of so-called generalized quantifiers as presented at the congress. In addition, some background information on logical language analysis, its possible applications and consequences is provided.

Vom 11.-16. Juli 1983 fand in Salzburg der 7. Internationale Kongreß für Logik, Methodologie und Wissenschaftstheorie statt. Mit über 500 Referaten in 14 Sektionen und 5 Symposien hatte dieser Kongreß außergewöhnliche Dimensionen. Insgesamt repräsentierte er jedoch nicht nur den Umfang, sondern vor allem auch die thematische Vielfalt der gegenwärtigen Diskussion in der Logik und Wissenschaftstheorie.

Die Themen der Symposien orientierten sich an aktuellen Problemen der Logik und Wissenschaftstheorie; sie lauteten im einzelnen:

- „Eine linguistische Wende: Neue Entwicklungen in der Logik“ (mit Charles Parsons, Vorsitzender, Jon Barwise und Johan van Benthem),

- „Die Struktur wissenschaftlicher Theorie“ (mit Wolfgang Stegmüller, I. Niiniluoto, J. D. Sneed und B. C. van Fraassen)

- „Leben und Werk Kurt Gödels“ (mit John W. Dawson Jr., Vorsitzender, S. Feferman, M. Baaz, E. Köhler, Ch. Thiel, M. Davis und J. W. Dawson Jr.),

- „Ethik der Medizin“ (mit Dag Prawitz, Vorsitzender, R. M. Hare und K. E. Tranøy)

- „Wissenschaftliche und ethische Rationalität“" (mit Evandro Agazzi, Vorsitzender, J. Ladrière und M. Przełeçki).

Die thematische Ausrichtung der 14 Sektionen richtete sich dagegen mehr auf die Grundlagen der Mathematischen und Philosophischen Logik, sowie die Grundlegung einzelner Wissenschaften. Auch die Themen der Sektionen seien im einzelnen aufgeführt: 
1. Beweistheorie und Grundlagen der Mathematik

2. Modelltheorie und ihre Anwendungen

3. Rekursionstheorie und Berechnungstheorie

4. Axiomatische Mengenlehre

5. Philosophische Logik

6. Allgemeine Methodologie der Wissenschaften

7. Grundlagen von Wahrscheinlichkeit und Induktion

8. Grundlagen und Philosophie der Naturwissenschaften

9. Grundlagen und Philosophie der Biologie

10. Grundlagen und Philosophie der Psychologie

11. Grundlagen und Philosophie der Sozialwissenschaften

12. Grundlagen und Philosophie der Linguistik

13. Geschichte der Logik, Methodologie und Wissenschaftstheorie

14. Grundprinzipien der Ethik der Wissenschaften.

In den einzelnen Sektionen wurden über die ganze Woche verteilt thematisch mehr oder weniger zusammengehörige Kurzreferate bzw. eingeladene Sektionsvorträge gehalten (mit nachfolgender Gelegenheit zur Diskussion), während die einzelnen Symposien ganztägig abgehalten wurden. Wer den Kongreß nicht nur besuchte, um zu sehen und/oder gesehen zu werden, dem bot sich daher mit dem Besuch eines Symposions die Gelegenheit zur eingehenden wissenschaftlichen Diskussion, ohne in dem Gedränge von Themen und Terminen der Sektionsvorträge zerrieben zu werden. Freilich: wer sich für den Besuch einer oder mehrerer Sektionen entschieden hatte, dem wird vielleicht auch aufgefallen sein, daß oft die interessantesten Vorträge - gewöhnlich vormittags - gleichzeitig angesetzt waren; hier wäre es vielleicht möglich gewesen, mit einer Verlängerung der täglichen Vortragszeit über $16 \mathrm{Uhr}$ hinaus, die besonders stark besuchten Sektionen 5, 6, 8, und 13, in denen jeweils bis zu drei Vorträge gleichzeitig stattfanden, zu entlasten.

$\mathrm{Da}$ es der Satz vom Widerspruch für jedermann - nicht nur für Logiker unmöglich macht, eine Veranstaltung zugleich zu besuchen und nicht zu besuchen, ist für eine detaillierte Berichterstattung eine thematische Beschränkung angesichts der Fülle und Vielfalt der Kongreßbeiträge unvermeidbar. Ich referiere im folgenden das Symposion der Sektion Philosophische Logik „Eine linguistische Wende: Neue Entwicklungen in der Logik," weil zum einen die rasante Entwicklung der logischen Sprachanalyse seit Montagues bahnbrechenden Arbeiten Anfang der 70er Jahre weiten Kreisen noch wenig vertraut sein dürfte, und zum anderen weil die Theorie verallgemeinerter Quantoren, um die es im wesentlichen geht, interessante und, wie ich meine, auch wichtige formale Neuerungen einführt, die grundlegende Fragen der logischen Anlayse aufwerfen und bis hin zu kognitionswissenschaftlichen Uberlegungen interessante Konsequenzen haben dürften.

Machen wir uns zunächst eine der Grundannahmen der MontagueGrammatik klar. Während für Frege die Logik (für die besonderen Zwecke, die sie erfüllen soll) der natürlichen Sprache überlegen ist, ist es eine Grundannahme der logischen Sprachanalyse Montagues, daß zwischen natürlichen und formalen Sprachen kein wesentlicher Unterschied besteht: natür- 
liche Sprachen lassen sich als formale Sprachen verstehen. Das ursprüngliche Ziel der logischen Sprachanalyse bzw. Formalen Semantik ist es daher, eine Theorie der Wahrheit für eine Sprache zu konstruieren, $d$. h. gesucht wird eine (rekursive) Definition der wahren Sätze einer Sprache und der in dieser Sprache geltenden Folgerungsbeziehungen. Unter Voraussetzung dieses Zieles, mit dem Prinzipien der Semantik formaler Sprachen auf die semantische Analyse natürlicher Sprachen angewendet werden, ist nun zu beachten, daß bei der Charakterisierung semantisch relevanter syntaktischer Strukturen verschiedene Strategien eingeschlagen werden können: im Extremfall hat man die Wahl zwischen einer einfachen Logik (etwa dem Prädikatenkalkül 1ter Stufe), welche eine strenge logische Kanonisierung und entsprechend komplexe Ubersetzungsregeln erfordert, oder einer komplexen Logik (etwa einem intensionalen Prädikatenkalkül 2ter Stufe), deren syntaktische Struktur dem syntaktischen Aufbau der natürlichen Sprache nachgebildet ist und entsprechend einfache Ubersetzungsregeln erfordert.

Betrachten wir zur Verdeutlichung der angeführten Gedanken ein Beispiel. Die traditionelle (wenngleich nicht aristotelische) Folgerungsbeziehung zwischen den Sätzen (1)-(3),

1. Alle Menschen sind sterblich,

2. Sokrates ist ein Mensch,

3. Sokrates ist sterblich, läßt sich auf verschiedene Weise formalisieren. Folgen wir der Fregeschen Formalisierung, dann drückt der Satz (1) die logische Beziehung der Unterordnung aus, die Sätze (2) und (3) dagegen die des Fallens eines Gegenstandes unter einen Begriff:
$1^{\prime}(\forall \mathrm{x})[\mathrm{M}(\mathrm{x}) \rightarrow \mathrm{St}(\mathrm{x})]$
$2^{\prime} \mathrm{M}(\mathrm{s})$$$
\begin{aligned}
\mathrm{M} & =\text { Mensch } \\
\mathrm{St} & =\text { sterblich } \\
\mathrm{s} & =\text { Sokrates }
\end{aligned}
$$
$3^{\prime} \mathrm{St}(\mathrm{s})$

In dieser Formalisierung divergiert jedoch die grammatische von der logischen Struktur: dem grammatischen Subjekt des Satzes (1) „Alle Menschen“, das, wie der Satz (3) zeigt, unter entsprechender Anpassung kongruierender Teile des Satzes durch einen Namen als Satzsubjekt ersetzt werden kann, fehlt in der Formulierung $\left(1^{\prime}\right)$ das logische Gegenstück. Als alternative Formulierung könnte die logische Formalisierung der Sätze (1)-(3) andererseits auch die grammatische Parralelität zwischen dem Subjekt des Satzes (1) „Alle Menschen" und der Sätze (2) und (3) ,Sokrates“ ausdrücken, d. h. an die Stelle der logischen Kanonisierung soll eine reichere Logik gesetzt werden, deren syntaktische Struktur dem syntaktischen Aufbau der natürlichen Sprache nachgebildet ist. Wie ließe sich dieser Ansatz durchführen? In seinem nunmehr klassischen Aufsatz „The Proper Treatment of Quantification in Ordinary English“ (1970/74) schlägt Montague vor, Eigennamen wie Quantoren zu behandeln (der umgekehrte Weg, Quantoren wie Eigennamen zu behandeln, führt bekanntlich zu absurden Konsequenzen); ist die Denotation des Quantors "Alle Menschen" die Menge von Eigenschaften, die alle 
Menschen haben, dann ist die entsprechende Denotation des Eigennamens „Sokrates" die Menge von Eigenschaften, die Sokrates hat. Für die logische Formalisierung ergibt sich daraus eine Umkehrung der Fregeschen Beziehungsverhältnisse: unter der vorgeschlagenen Interpretation von Quantoren und Eigennamen ist der Satz (1) bzw. (3) wahr, wenn die Eigenschaft sterblich zu sein eine der Eigenschaften ist, die allen Menschen bzw. Sokrates zukommt. Im Kalkül der Montague-Grammatik erhalten wir dementsprechend als logische Analyse der Sätze (1)-(3) die folgende Formalisierungen (vereinfacht):

$$
\begin{array}{rlrl}
1^{\prime \prime}(\lambda F)[\nabla x)(M(x) \rightarrow F(x)] . S t & M & =\text { Mensch } \\
2^{\prime \prime}(\lambda F) F(s) . M & F & =\text { Prädikatsvariable } \\
& & S t & =\text { sterblich } \\
3^{\prime \prime} & (\lambda F) F(s) . S t & s & =\text { Sokrates }
\end{array}
$$

(Die Grundregel des $\lambda$-Kalküls lautet: $(\lambda x) F(x) . a \rightarrow F(a)$.)

Stand in der 1. Phase der Entwicklung der Montague-Grammatik zunächst die Ausarbeitung der formalen Analyse bestimmter Konstruktionen, wie z. B. von Tempus, der Modalitäten oder der Komparative, im Vordergrund, so etwa in den Arbeiten von Kamp (1979) und Kratzer (1981), so zeigen die neueren Arbeiten der 2. Phase der Entwicklung ein verstärktes Interesse an bestimmten semantischen Kategorien, insbesondere an der Kategorie der sog. Determinatoren, und den semantischen Beschränkungen, welche den tatsächlichen linguistischen Inhalt dieser Kategorien bestimmen (vgl. den von van der Auwera herausgegebenen Sammelband „The Semantics of Determiners" und den Aufsatz "Generalized Quantifiers and Natural Language“ von Jon Barwise und Robin Cooper (1981)). Die neuesten Arbeiten im Bereich der formalen Semantik, die man als die 3. Entwicklungsphase bezeichnen könnte, versuchen, die Dynamik und Kontextabhängigkeit der Interpretation in den Analyserahmen der logischen Sprachanalyse zu integrieren, wie z. B. Ballmer (1978) und Barwise und Perry (1983, soeben erschienen). Jon Barwise, der leider nicht persönlich anwesend war und dessen Beitrag von Hans Kamp verlesen wurde, stellte in seinem Symposions-Vortrag "The Situation in Logic" seine neueren Uberlegungen zu einer auf dem Informationsbegriff aufbauenden Situations-Logik vor. Der Vortrag von Johan van Benthem „A Linguistic Turn: New Directions in Logic", der die im folgenden darzustellende Theorie verallgemeinerter Quantoren aufgreift, gehört dagegen eher zur 2. Phase.

Die mathematische Theorie verallgemeinerter Quantoren geht auf Mostowski zurück (Mostowski, 1957; vgl. u. a. Bell-Slomson, 1969); eine Darstellung im Formalismus der Montague-Grammatik und eine Anwendung auf die natürliche Sprache findet sich in dem oben erwähnten Aufsatz von Barwise und Cooper (1981), wo unter der Denotation eines QuantorenAusdrucks - wie oben angedeutet - eine Menge von Eigenschaften, d. h. eine Menge von Mengen verstanden wird. (Ein Quantoren-Ausdruck wie z. B. die normalsprachlichen Ausdrücke "alle", "die meisten", ,, wenige“, „kein", usw., ergeben in Kombination mit einem Nomen einen Quantor.) Anders als Barwise und Cooper faßt van Benthem einen Quantoren-Ausdruck jedoch als Relation auf; ein verallgemeinerter Quantoren-Ausdruck wird damit als ein 
Funktor $Q$ aufgefaßt, der in einem gegebenen universe of discourse $E$ eine $z w e i s t e l l i g e$ Relation $Q_{E}$ zwischen Teilmengen von $E$ definiert, z. B.

([Alle] $\mathrm{AB})=$ wahr dann und nur dann, wenn $\mathrm{A} \subseteq \mathrm{B}$

([Die meisten $] A B)=$ wahr dann und nur dann, wenn $|A \cap B|>|A-B|$.

Unter Beschränkung auf endliche Gegenstandsbereiche sollen nun allgemeine Bedingungen angegeben werden, welche von Quantoren-Ausdrücken der natürlichen Sprache erfüllt werden. Die linguistische Wende, von der in dem gleichnamigen Symposion die Rede war, läßt sich damit in dreierlei Hinsicht präzisieren: erstens in der für die Montague-Grammatik charakteristischen, linguistisch motivierten Absicht, die logischen Strukturen den grammatischen Strukturen anzunähern; zweitens in der Erweiterung des Analysebereichs der logischen Sprachanalyse, der nunmehr auch die Analyse sog. nicht-logischer Quantoren-Ausdrücke der natürlichen Sprache, wie z. B. "die meisten", „viele“, „mehr als die Hälfte“ usw., umfaßt, die bekanntlich nicht im Rahmen des Prädikatenkalküls 1ter Stufe definiert werden können; und drittens in dem Gedanken, durch die Analyse traditioneller logischer Kategorien allgemeine Bedingungen zu erhalten, welche die Formulierung semantischer Universalien zu erlauben scheinen. Wie sich zeigen wird, geht die Ausführung dieser Ziele jedoch über eine bloß ,linguistische Wende" in der Logik hinaus und eröffnet weitergehende Perspektiven, als der programmatische Titel vermuten läßt.

Unter der Voraussetzung, daß ein Quantoren-Ausdruck eine Relation zwischen Mengen definiert, lassen sich die folgenden Grundbedingungen von (logischen und nicht-logischen) Quantoren-Ausdrücken angeben.

\section{Konservativität}

Für alle $E, A, B \subseteq E$,

$Q_{E} A B$ dann und nur dann, wenn $Q_{E} A(B \cap A)$.

M.a.W., der Quantoren-Ausdruck „lebt" von dem ersten Argument; um den ganzen quantifizierten Satz zu bewerten, braucht man lediglich die von dem Nomen A denotierte Menge zu betrachten.

\section{Extension}

Für alle $E^{\prime} \subseteq E, A, B \subseteq E^{\prime}$,

$\mathrm{Q}_{E^{\prime}} \mathrm{AB}$ dann und nur dann, wenn $\mathrm{Q}_{\mathrm{E}} \mathrm{AB}$.

D. h., das kleinere universe of discourse $E^{\prime}$ kann stets zugunsten eines größeren vernachlässigt werden.

\section{Quantität}

Für alle $\mathrm{E}, \mathrm{A}, \mathrm{B} \subseteq \mathrm{E}$, und $\phi$ eine bijektive Abbildung in $\mathrm{E}$, $\mathrm{Q}_{E} \mathrm{AB}$ dann und nur dann, wenn $\mathrm{Q}_{\phi(\mathrm{E})} \phi(\mathrm{A}) \phi(\mathrm{B})$.

Wird die Menge $A$ bijektiv auf die Menge $A^{\prime}$ und die Menge $B$ bijektiv auf die Menge $B^{\prime}$ abgebildet, dann wird auch der Durchschnitt der Mengen A und B bijektiv auf den Durchschnitt der Mengen $A^{\prime}$ und B' abgebildet; die Bedingung der Quantität garantiert, daß für die Bewertung eines quantifizierten Satzes lediglich die Kardinalität von $|A \cap B|$, $|A-B|,|B-A|$ und $|E-(A \cup B)|$ berücksichtigt werden muß. M.a.W., ein Quantoren-Ausdruck verhält sich gegenstandsneutral und berücksich- 
tigt lediglich die Anzahl von Individuen in der von seinen Argumenten denotierten Mengen.

Die mit diesen Grundbedinqungen charakterisierte relationale Auffassung der Quantoren-Ausdrücke erlaubt es, Quantoren-Ausdrücke in einem,Zahlenbaum' darzustellen, $d$. $h$. allen Paaren $\langle a, b\rangle$, derart daß $a=|A-B|$ und $b=|A \cap B|$ bezüglich steigender Kardinalität von $A$, wobei gilt $|B| \geqslant \mid A]$ :

$$
\begin{aligned}
& |\mathrm{A}|=0 \\
& |\mathrm{~A}|=1 \\
& |\mathrm{~A}|=2 \\
& |\mathrm{~A}|=3 \\
& |\mathrm{~A}|=4
\end{aligned}
$$

$$
\begin{aligned}
& 0,0 \\
& \begin{array}{lll}
1,0 & 0,1
\end{array} \\
& 2,0 \quad 1,1 \quad 0,2 \\
& \begin{array}{llll}
3,0 & 2,1 & 1,2 & 0,3
\end{array} \\
& \begin{array}{lllll}
4,0 & 3,1 & 2,2 & 1,3 & 0,4
\end{array} \\
& \text { usw. }
\end{aligned}
$$

Ein Quantoren-Ausdruck läßt sich nun mittels der Menge von Paaren definieren, auf die er zutrifft; so entspricht z. B. dem Quantoren-Ausdruck „Alle“ in der folgenden Darstellung die schräg gestreifte Fläche am äußersten rechten Rand, dem Quantoren-Ausdruck „Kein" dagegen die schräg gestreifte Fläche am äußersten linken Rand; die dazwischenliegende Fläche wird von den Quantoren-Ausdrücken „Nicht alle" und „einige" belegt:

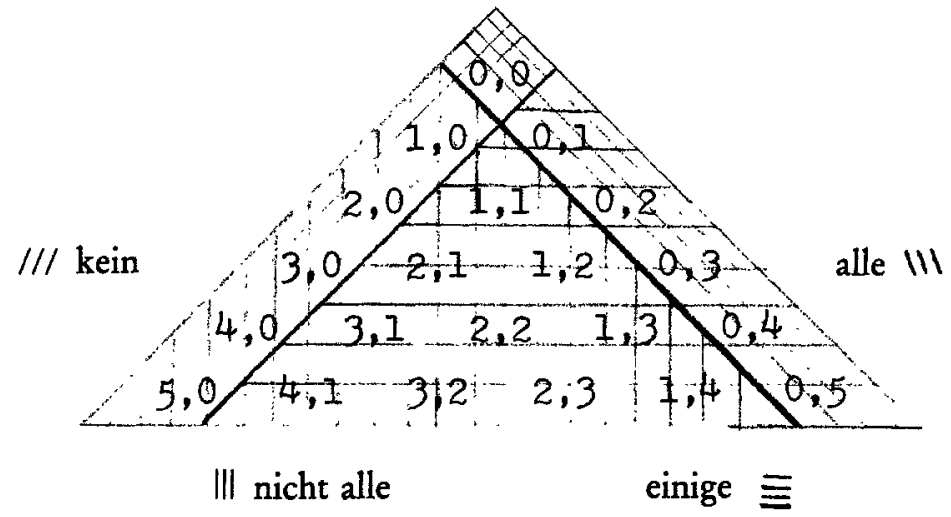

Als weitere Bedingungen für Quantoren-Ausdrücke lassen sich formulieren:

IV. Verschiedenbeit

Für alle $\mathrm{E}, \emptyset \neq \mathrm{A} \subseteq \mathrm{E}$ gibt es ein $\mathrm{B}, \mathrm{B}^{\prime} \subseteq \mathrm{E}$, derart daß $\mathrm{Q}_{\mathrm{E}} \mathrm{AB}$, aber nicht $\mathrm{Q}_{\mathrm{E}} \mathrm{AB}^{\prime}$.

Jeder ,vernünftige“ Quantoren-Ausdruck belegt also nicht alle Paare im Zahlenbaum, d.h. jede Zeile $\geqslant 2$ enthält sowohl Paare, auf die der Quantoren-Ausdruck zutrifft, als auch Paare, auf die er nicht zutrifft.

\section{Monotonie}

Ein Quantoren-Ausdruck ist rechts-monoton steigend (MON $\uparrow)$, wenn gilt:

$\mathrm{Q}_{\mathrm{E}} \mathrm{AB}, \mathrm{B} \subseteq \mathrm{B}^{\prime}$ impliziert $\mathrm{Q}_{\mathrm{E}} \mathrm{AB}^{\prime} ;$ 
ein Quantoren-Ausdruck ist rechts-monoton fallend (MON $\downarrow$ ), wenn gilt:

$\mathrm{Q}_{\mathrm{E}} \mathrm{AB}, \mathrm{B}^{\prime} \subseteq \mathrm{B}$ impliziert $\mathrm{Q}_{\mathrm{E}} A \mathrm{~B}^{\prime}$;

ein Quantoren-Ausdruck ist links-monoton steigend ( $\uparrow \mathrm{MON})$, wenn gilt:

$\mathrm{Q}_{\mathrm{E}} \mathrm{AB}, \mathrm{A} \subseteq \mathrm{A}^{\prime}$ impliziert $\mathrm{Q}_{\mathrm{E}} \mathrm{A}^{\prime} \mathrm{B}$;

ein Quantoren-Āusdruck ist links-monoton fallend ( $\downarrow M O N)$, wenn gilt: $\mathrm{Q}_{\mathrm{E}} \mathrm{AB}, \mathrm{A}^{\prime} \subseteq \mathrm{A}$ impliziert $\mathrm{Q}_{\mathrm{E}} \mathrm{A}^{\prime} \mathrm{B}$.

Betrachten wir bestimmte linguistische Substitutionen, dann erhalten wir für das Deutsche u. a. die folgenden Klassifikationen:

rechts-monoton steigend: alle, einige, viele, wenigstens zwei;

rechts-monoton fallend: kein, keiner, keiner von beiden, höchstens zwei;

links-monoton steigend: einige, nicht alle, wenige;

links-monoton fallend: alle, kein, viele;

nicht monoton: genau zwei, genau die Hälfte. ${ }^{1}$

Insbesondere für die Quantoren-Ausdrücke des logischen Quadrates gelten also folgende Monotonie-Bedingungen:

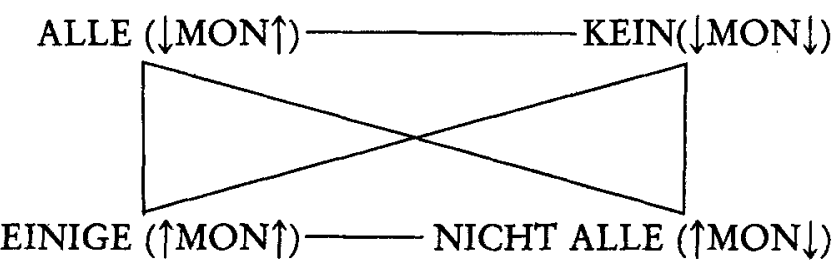

Als zweistellige Relationen gelten für Quantoren-Ausdrücke natürlich auch alle bekannten Relationseigenschaften, wie Reflexivität, Symmetrie, Transitivität, Linearität usw., wobei zu beachten ist, daß die Relationseigenschaften von Quantoren-Ausdrücken deren Verhalten in Folgerungszusammenhängen charakterisieren; ohne diese Bedingungen im einzelnen anzuführen, seien als Beispiel wieder die Quantoren-Ausdrücke des logischen Ouadrates angegeben:

Alle: reflexiv und transitiv

Einige : symmetrisch und quasi-reflexiv $(\forall x y)(R(x, y) \rightarrow R(x, x))$

Kein: symmetrisch und quasi-universal $(\forall x y)(R(x, x) \rightarrow R(x, y))$

Nicht alle: irreflexiv und linear.

Obwohl die genannten Bedingungen nur eine kleine Auswahl aus den formulierbaren Bedingungen darstellen, - für eine ausführliche Darstellung und Definition dieser und anderer Bedingungen sei auf die Arbeiten von Barwise und Cooper (1981), sowie van Benthems (1983) verwiesen -, so

${ }^{1}$ Für Quantoren-Ausdrücke wie „viele“ und „wenige“ muß offenbar zwischen einer ,absoluten und ,relativen' Bedeutung unterschieden werden: auch wenn vielleicht relativ zur Gesamtzahl von Philosophen gilt, daß viele Philosophen weise sind, dann braucht doch lange nicht $\mathrm{zu}$ gelten, daß auch die Zahl der weisen Menschen absolut gesehen groß ist. 
erlauben sie doch im Sinne der gestellten Frage nach möglichen semantischen Universalien u. a. folgende Hypothesen:

SU1: Alle natürlichen Sprachen enthalten Quantoren-Ausdrücke, welche die Bedingung der Konservativität erfüllen.

SU2: Alle definierten Quantoren-Ausdrücke einer natürlichen Sprache erfüllen die Bedingung der Verschiedenheit, $d . h$. alle definierten Quantoren-Ausdrücke verhalten sich wie Filter.

SU3: Keine natürliche Sprache hat asymmetrische Quantoren-Ausdrücke.

SU4: Keine natürliche Sprache hat ,zirkuläre' Quantoren-Ausdrücke, derart daß gilt: QXY, QYZ $\rightarrow$ QZX.

Die Theorie verallgemeinerter Quantoren liefert jedoch auch Ergebnnisse, welche die Logik selber betreffen; so formuliert van Benthem u. a. die folgenden Theoreme:

TH1:Das logische Quadrat besteht aus genau den links-monotonen logischen Quantoren.

Th2: Links-Monotonie impliziert Definierbarkeit im Prädikatenkalkül 1 ter Stufe.

Wurde in der traditionellen Logik die Existenz logischer Konstanten oft als ein unhinterfragbares Faktum angesehen, so scheint die Theorie verallgemeinerter Quantoren eine Erklärung und allgemeine Charakterisierung logischer Konstanten zu ermöglichen, z. B. für die Quantoren-Ausdrücke des Logischen Quadrats und ihre zentrale Stellung in der traditionellen Logik. Es tut sich damit für die Logik eine Forschungsstrategie auf, die bisher wenig beachtet worden ist: anstatt, wie bisher üblich, von einer bestimmten Menge logischer Konstanten auszugehen und zu fragen, welche logischen Folgerungen sie zulassen, könnte man die zulässigen Schlußschemata einer Theorie, z. B. der Syllogistik, betrachten und fragen, welche logischen Konstanten, z. B. welche Quantoren-Ausdrücke, diese Theorie erfüllen könnten.

Wohin führt uns also die linguistische Wende in der Logik? Handelt es sich lediglich um eine "linguistische" Wende, die allein für Linguisten und formale Semantiker von Interesse ist? Der Titel des Symposions „Eine linguistische Wende: Neue Entwicklungen in der Logik" scheint mir symptomatisch zu sein für ein erweitertes Verständnis von Logik, welches sich auch in den einzelnen Sektionsbeiträgen zeigte und insgesamt ein wachsendes Interesse an kognitiven Fragestellungen (,Wie denken wir?") und an der Geschichte der Logik erkennen lie $\beta^{2}$. Hat sich die moderne Logik in ihren Anfängen unter dem Einfluß Freges vor allem an der Mathematik orientiert, so ist inzwischen vielleicht die Zeit gekommen für eine Rückbesinnung auf die Vor-Fregesche Tradition der Logik: auf die natürliche Sprache als Quelle logischer Inspiration.

\footnotetext{
2 Cf. u. a. die kürzlich erschienenen Bücher von Fred Sommers "The Logic of Natural Language" (1982) und Phillipp Johnson-Laird „Mental Models" (1983).
} 


\section{BIBLIOGRAPHIE}

van der Aufwera, J. (ed.) (1980), The Semantics of Determiners, London.

Bäuerle, R. et al. (eds.) (1979), Semantics from different points of view. Berlin.

Ballmer, Th. (1978), Logical Grammar, Amsterdam.

Barwise, J. und R. Cooper (1981), Generalized Quantifiers and Natural Language, Linguistics and Philosophy 4, S. 159-219.

Barwise, J. und J. Perry (1983), Situations and Attitudes, Montgomery.

Bell, J. L. und A. B. Slomson (1969), Models and Ultraproducts - An Introduction, Amsterdam. van Benthem, J. (1983a), A linguistic turn: new directions in logic - An Outline, Fotokopie der Gliederung des Salzburger Vortrags, Salzburg.

- (1983b), Determiners and Logic, Linguistics and Philosophy 6, S. 447-478.

- (1983c), Questions about Quantifiers, Journal of Symbolic Logic, erscheint demnächst.

Cooper, R. (1983), Quantification and Syntactic Theory, Dordrecht.

Eikmeyer, H.-J. und M. Rieser (eds.) (1981), Words, Worlds, and Context, Berlin.

Heyer, G. (1983), Generische Kennzeichnungen. Zur Logik und Ontologie generischer Bedeutung, Dissertation, Philosophia Verlag München und Wien, erscheint demnächst.

Johnson-Laird, P. (1983), Mental Models, Cambridge (Mass.).

Kamp, H. (1979), Instants, Events, and Temporal Discourse, in: R. Bäuerle et. al. (eds.) (1979).

Kratzer, A. (1981), The Notional Category of Modality, in: H.-J. Eikmeyer und H. Rieser (eds.) (1981).

Montague, R. (1974), Formal Philosophy. Selected Papers of Richard Montague, hrsg. von R. Thomason, New Haven.

- (1970/1974), The Proper Treatment of Quantification in Ordinary English, in: R. Montague (1974).

Mostowski, A. (1957), On a Generalization of Quantifiers, Fundamenta Mathematica 44, S. 12-36.

Schnelle, H. (1973), Sprachphilosophie und Linguistik, Reinbeck b. Hamburg.

Sommers, F. (1982), The Logic of Natural Language, Oxford.

Adresse des Autors:

Dr. Gerhard Heyer, University of Michigan, Dept. of Linguistics, 1076 Frieze, Ann Arbor, Mi. $48109 /$ USA 